SHORT REPORT

\title{
Subthalamic nucleus stimulation in tremor dominant parkinsonian patients with previous thalamic surgery
}

\author{
V Fraix, P Pollak, E Moro, S Chabardes, J Xie, C Ardouin, A L Benabid
}

J Neurol Neurosurg Psychiatry 2005;76:246-248. doi: 10.1136/jnnp.2003.022707

Before the introduction of high frequency stimulation of the subthalamic nucleus (STN), many disabled tremor dominant parkinsonian patients underwent lesioning or chronic electrical stimulation of the thalamus. We studied the effects of STN stimulation in patients with previous ventral intermediate nucleus (VIM) surgery whose motor state worsened. Fifteen parkinsonian patients were included in this study: nine with unilateral and two with bilateral VIM stimulation, three with unilateral thalamotomy, and one with both unilateral thalamotomy and contralateral VIM stimulation. The clinical evaluation consisted of a formal motor assessment using the Unified Parkinson's Disease Rating Scale (UPDRS) and neuropsychological tests encompassing a 50 point frontal scale, the Mattis Dementia Rating Scale, and the Beck Depression Inventory. The first surgical procedure was performed a mean (SD) of 8 (5) years after the onset of disease. STN implantation was carried out 10 (4) years later, and duration of follow up after beginning STN stimulation was 24 (20) months. The UPDRS motor score, tremor score, difficulties in performance of activities of daily living, and levodopa equivalent daily dose significantly decreased after STN stimulation. Neither axial symptoms nor neuropsychological status significantly worsened after the implantation of the STN electrodes. The parkinsonian motor state is greatly improved by bilateral STN stimulation even in patients with previous thalamic surgery, and STN stimulation is more effective than VIM stimulation in tremor dominant parkinsonian patients.

B efore the introduction of deep brain stimulation (DBS), many patients with tremor dominant Parkinson's disease (PD), severely disabled in spite of all available drug strategies, underwent a unilateral lesion of the ventral intermediate nucleus (VIM) of the thalamus. ${ }^{1}$ Bilateral procedures were generally avoided in the belief that they had disabling adverse effects such as dysarthria, postural instability, or cognitive impairment. Since the introduction of DBS, bilateral procedures have been performed more safely, but many patients with asymmetrical motor impairment continued to be operated on unilaterally. Some of these secondarily developed contralateral parkinsonian symptoms or motor fluctuations, and levodopa induced dyskinesia. $^{23}$ Moreover, reduction of tremor was often incomplete or could extend to the other ipsilateral limb..$^{3-5}$ Because bilateral subthalamic nucleus (STN) stimulation has proved to be effective on levodopa responsive motor symptoms, ${ }^{67}$ we studied its effects in patients with previous VIM surgery who were disabled either because of recurrence or worsening of tremor or levodopa induced motor complications.

\section{PATIENTS AND METHODS}

Fifteen parkinsonian patients who had undergone VIM surgery ( 11 in our centre and four in other centres) were included in this study. They were bilaterally implanted in the STN, except for one patient who underwent unilateral STN stimulation. Their mean (SD) age at onset of disease was 42 (6) years and the first operation was performed 8 (5) years later. The main clinical features of patients at onset of PD, data on the VIM and STN surgical procedures, and type of implanted lead are reported in table 1 . Patients who underwent VIM DBS between 1987 and 1991 had monopolar leads (SP-5535, currently 3388 series; Medtronic, Minneapolis, USA). Since 1992, we have used quadripolar leads (Model 3387 or 3389 DBS; Medtronic). The mean (SD) coordinates of the most distal contact of the 14 implanted leads were $6.30(1.78) \mathrm{mm}$ anterior to the posterior commissure, 0.05 $(0.88) \mathrm{mm}$ below the anterior-posterior commissure line, and 14.16 (1.24) $\mathrm{mm}$ lateral to midline. The thalamic lesions were located 11.5 (4.1) mm lateral to midline. Although we lack detailed clinical results concerning the patients from other centres, all were initially improved by VIM surgery and one had a mild contralateral hemiparesis after thalamotomy (patient 3). In patient 2, tremor recurred within a few months after lesioning. In patients 8 and 10 with VIM stimulation, the initial decrease in upper limb rest tremor was subsequently lost before STN surgery. Patients 7, 12, and 15 showed little benefit from VIM stimulation, with a 1 point decrease in contralateral upper or lower limb rest tremor subscore of the Unified Parkinson's Disease Rating Scale (UPDRS). Seven of the 12 patients with VIM DBS (patients 4 , $6,9,11,13,14$, and 15) had at least a 2 point decrease in rest tremor subscore on the arm but secondarily developed ipsilateral lower limb rest tremor not improved by DBS. All patients but one also developed disabling motor fluctuations and levodopa induced dyskinesia, a mean (SD) of 8.5 (3.4) and 9.9 (4.4) years respectively after PD onset. STN surgery was performed 10 (4) years after VIM surgery (range 314 years after VIM DBS, 8-19 years after lesioning). The surgical procedure has been described previously. ${ }^{6}$ The thalamic leads were disconnected from the implanted pulse generator, but not removed.

The motor state was non-blindly assessed with the UPDRS part III. ${ }^{8}$ Rest (item 20, maximum score 20) and postural and action tremor (item 21, maximum score 8) were scored separately. Axial symptoms, including speech (item 18), postural stability (item 30) and gait (item 29) were also scored separately. Disability in daily living was assessed with the UPDRS part II, and dyskinesia duration and disability with the UPDRS part IV (items 32 and 33). The levodopa

\footnotetext{
Abbreviations: DBS, deep brain stimulation; LEDD, levodopa equivalent daily dose; PD, Parkinson's disease; PDQL-37, Parkinson's Disease Quality of Life 37 point scale; STN, subthalamic nucleus; UPDRS, Unified Parkinson's Disease Rating Scale; VIM, ventral intermediate nucleus
} 
Table 1 Patients' characteristics, indication, type and material for VIM and STN surgical procedures

\begin{tabular}{|c|c|c|c|c|c|c|c|c|}
\hline Patient & $\begin{array}{l}\text { Age at PD } \\
\text { onset } \\
\text { (years) }\end{array}$ & $\begin{array}{l}\text { PD duration } \\
\text { before VIM } \\
\text { (years) }\end{array}$ & $\begin{array}{l}\text { Indications } \\
\text { for VIM }\end{array}$ & VIM surgery & $\begin{array}{l}\text { VIM DBS } \\
\text { lead }\end{array}$ & $\begin{array}{l}\text { Interval } \\
\text { between } \\
\text { VIM and } \\
\text { STN (years) }\end{array}$ & $\begin{array}{l}\text { Indication for STN } \\
\text { DBS (lead 3389) }\end{array}$ & Side \\
\hline 1 & 32 & 5 & L UL tremor & $\mathrm{R}$ thalamotomy & - & 19 & MF & $\mathrm{Bi}$ \\
\hline 2 & 34 & 8 & L UL tremor & $\mathrm{R}$ thalamotomy & - & 12 & $\mathrm{Bi}$ UL \& LL T+MF & $\mathrm{Bi}$ \\
\hline 3 & 32 & 19 & R UL tremor & L thalamotomy & - & 8 & MF & $\mathrm{Bi}$ \\
\hline \multirow[t]{2}{*}{4} & 46 & 2 & R UL tremor & $\mathrm{L}$ thalamotomy & - & - & & \\
\hline & 46 & 10 & L UL tremor & R VIM DBS & 3387 & 7 & L LL tremor & $\mathrm{R}$ \\
\hline 5 & 53 & 3 & L UL tremor & R VIM DBS & Monopolar & 14 & Bi UL \& LL T & $\mathrm{Bi}$ \\
\hline 6 & 47 & 6 & R UL tremor & L VIM DBS & Monopolar & 10 & MF & $\mathrm{Bi}$ \\
\hline 7 & 40 & 12 & R UL tremor & Bilateral VIM DBS & Monopolar & 11 & bilat. UL \& LL T+MF & $\mathrm{Bi}$ \\
\hline 8 & 40 & 9 & L UL tremor & Bilateral VIM DBS & Monopolar & 12 & L UL \& LL T+MF & $\mathrm{Bi}$ \\
\hline 9 & 38 & 8 & L UL tremor & $\mathrm{R}$ vim DBS & 3387 & 6 & MF & $\mathrm{Bi}$ \\
\hline 10 & 39 & 9 & R UL tremor & L VIM DBS & 3387 & 5 & $\mathrm{Bi}$ UL \& LL T+MF & $\mathrm{Bi}$ \\
\hline 11 & 48 & 14 & L UL tremor & R VIM DBS & 3387 & 8 & $\mathrm{~L} \mathrm{LL} \mathrm{T+MF}$ & $\mathrm{Bi}$ \\
\hline 12 & 46 & 4 & R LL tremor & L VIM DBS & Monopolar & 8 & R UL T+MF & $\mathrm{Bi}$ \\
\hline 13 & 50 & 14 & R UL tremor & L VIM DBS & 3387 & 3 & L LL T+MF & $\mathrm{Bi}$ \\
\hline 14 & 46 & 2 & L UL tremor & R VIM DBS & Monopolar & 12 & L LL T+MF & $\mathrm{Bi}$ \\
\hline 15 & 43 & 1 & R UL tremor & L VIM DBS & Monopolar & 11 & MF & $\mathrm{Bi}$ \\
\hline
\end{tabular}

equivalent daily dose (LEDD) was calculated as previously reported. ${ }^{9}$ The impact of STN surgery on quality of life was evaluated with a health specific scale, the Parkinson's Disease Quality of Life (PDQL-37) scale. ${ }^{10}$ Cognitive function was assessed with the Mattis Dementia Rating Scale and a 50 point global frontal scale, and mood with the Beck Depression Inventory. ${ }^{11}$ The results are reported as means (SD). The data of patients with VIM lesion were pooled with that of the VIM DBS on stimulation subgroup, as electrical stimulation is considered to mimic the effects of lesioning. Because of the small size of our series, we performed a non-parametric statistical analysis on all the above parameters, using the Wilcoxon signed rank test, to assess the effects of STN stimulation. A p value of 0.05 was considered statistically significant.

\section{RESULTS}

The mean (SD) follow up after STN implantation was 24 (20) months. The effects of VIM and STN surgery on the UPDRS total motor score, rest and postural tremor subscores in off medication condition are reported in fig 1. Before STN implantation, VIM DBS did not decrease the UPDRS total motor score, although the rest tremor score did decrease significantly. Subthalamic nucleus stimulation reduced the UPDRS total motor score and, in seven patients (patients 1, 2, $3,8,9,11$, and 13), abolished postural and rest tremor in the off medication and on stimulation condition. Before STN implantation, in the off medication condition, VIM stimulation did not modify rigidity, akinesia, postural stability, or gait. With STN stimulation, rigidity decreased from 6.8 (3.9) to $2.7(2.7)(\mathrm{p}<0.01)$, akinesia from $14.6(6.6)$ to $6.7(2.7)$ $(\mathrm{p}<0.002)$, postural stability from $1.2(0.6)$ to $0.8(0.8)$ $(\mathrm{p}<0.02)$, and gait from $1.4(0.7)$ to $0.6(0.6)(\mathrm{p}<0.001)$. The rating of speech did not change with STN DBS in the off medication condition. In the on medication condition, the UPDRS part IV (items 32 and 33) decreased from 2.6 (2.0) under VIM stimulation to 0.6 (1.2) under STN stimulation $(p<0.001)$. No change in behaviour or cognition occurred after STN surgery. Quality of life improved, and disability was reduced after STN stimulation. The PDQL-37 score increased from $103(20)$ to $136(20)(\mathrm{p}<0.01)$, and the UPDRS part II decreased from 27.3 (6.7) to $9.7 \quad(6.3) \quad(p<0.001)$. The improvement in motor symptoms after STN stimulation allowed LEDD to be reduced from 1203 (491) mg/day to 315 (258) $\mathrm{mg} /$ day $(\mathrm{p}<0.001)$. No serious adverse event occurred during the STN surgical procedure. During the setting of the electrical parameters, we observed hemifacial contractions with eight leads, upper limb paresthesia with seven, diplopia with six, dyskinesias with two, dysarthria with two, dizziness with one, body warmness with two, and no response with one. These side effects are similar to those usually reported; chronic electrical parameters were set at not more than $90 \%$ of the threshold for permanent side effects. ${ }^{7}$
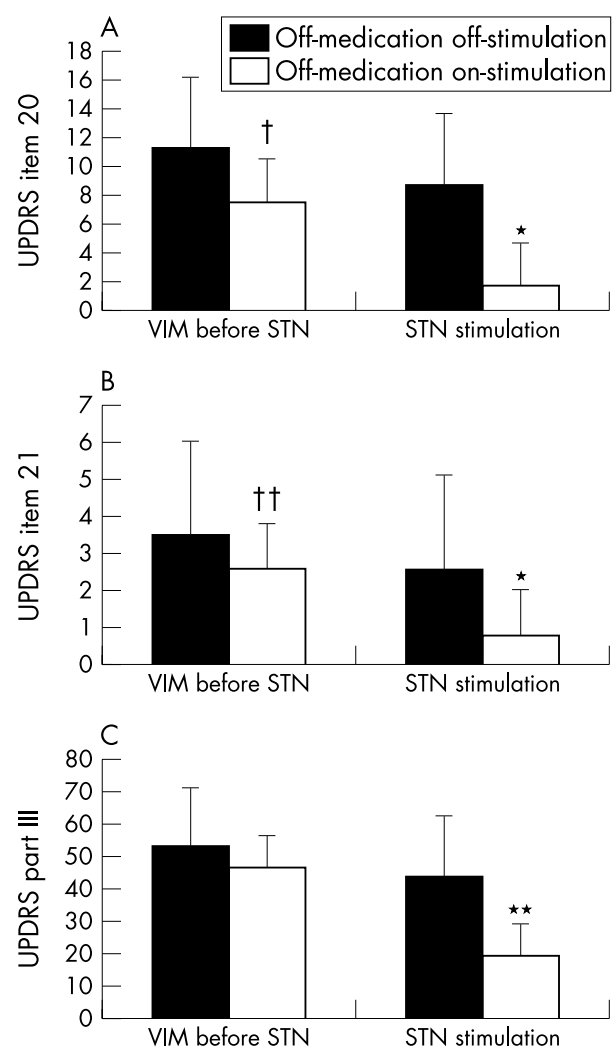

Figure 1 UPDRS motor examination: (a) rest tremor score (item 20); (b) postural tremor score (item 21), total motor score (UPDRS part III); and (c) VIM stimulation before STN DBS $(n=12)$ and after STN stimulation in off medication condition $(n=15)$. Filled squares: off medication, off stimulation; open squares: off medication, on stimulation. ${ }^{*} p<0.005$ for the comparison with the off medication VIM stimulation condition before STN stimulation; ${ }^{* *} p<0.001$ for the comparison with the off medication, VIM stimulation condition before STN stimulation; $t p<0.02$ for the comparison with the off medication, off stimulation condition; $\uparrow \dagger p<0.01$ for the comparison with the off medication, off stimulation condition. 


\section{DISCUSSION}

Fifteen severely disabled parkinsonian patients underwent STN stimulation because of a lack or loss of effect of previous VIM surgery over time associated with tremor worsening or spreading to other limbs, or levodopa induced motor complications. As all patients had a relatively young onset of PD, and 11 underwent VIM surgery in the same centre, long term follow up was detailed. After VIM surgery, contralateral rest tremor was almost totally suppressed. These results are similar to those previously published. ${ }^{12-14}$ Improvement in contralateral rest limb tremor could remain unchanged over more than 6 years and up to 19 years. ${ }^{23}$ As described in about $20 \%$ of cases, recurrence of tremor occurred in one patient after staged VIM thalamotomy, due to a lesion that was too medial according to the Guiot diagram. ${ }^{5}$ As the other VIM lesions and leads were correctly placed, ${ }^{15}$ the loss of effect of VIM DBS described in two patients might be due to worsening of rest and postural limb tremor. All patients but one secondarily developed lower limb tremor on which VIM surgery had very little effect. ${ }^{5}$ As the patients were relatively young with a tremor dominant form at onset of PD, they secondarily developed rigidity, akinesia, and motor complications, on which the effects of VIM surgery were poor. ${ }^{3}$ Even though long term VIM stimulation remained effective on tremor, the improvement in the patients' ability to cope with daily living was variable. ${ }^{16} 17$

Disease progression and motor complications might explain the recurrence of disability, and the development of dependence on a caregiver for one or more activities of daily living within 10 years after VIM surgery. The decrease in LEDD and the improvement in motor symptoms and quality of life after STN stimulation were similar to those reported in patients given surgery for the first time..$^{67^{18-20}}$ The effect of STN stimulation on rest and postural tremor has been reported to be stable over time..$^{21} 22$ Considering the reported effects of bilateral VIM surgery on speech and postural stability, we feared that implanting leads bilaterally in a second target would worsen axial symptoms. However, as reported in both non-operated patients, and patients with previous surgery, these symptoms improved after bilateral STN stimulation. ${ }^{23-26}$ The outcome concerning the neuropsychological status confirmed that there was no cognitive deterioration after STN DBS in those patients with young onset PD and no preoperative impairment, although they underwent surgery in four brain areas. ${ }^{11} 2728$ So far, no patient with STN stimulation has needed to be secondarily implanted in the VIM because of a lack or loss of effect. In this study, the overall improvement after STN implantation was clearly better than after VIM surgery. This study confirms that STN stimulation shows benefits on parkinsonian tremor in the long term. Hence, this procedure could be used to treat young onset PD patients, including those with the tremor predominant type. Parkinsonian patients with VIM lesions or DBS may benefit from STN stimulation, especially if they develop levodopa induced motor complications or tremor recurrence.

\section{Authors' affiliations}

V Fraix, P Pollak, E Moro, J Xie, C Ardouin, Department of Neurology, University Hospital of Grenoble, France

V Fraix, P Pollak, E Moro, S Chabardes, J Xie, C Ardouin, A L Benabid, INSERM U318, Joseph Fourier University, Grenoble, France

S Chabardes, A L Benabid, Department of Neurosurgery, University of Grenoble, France

Competing interests: $\mathrm{P}$ Pollak received reimbursment for attending symposia and once a fee for speaking from Medtronic. A-L Benabid received reimbursment for attending symposia, and funds for research.
Correspondence to: Dr V Fraix, Department of Neurology, Grenoble University Hospital, BP 217, 38043 Grenoble cedex 9, France; valerie. fraix@ujf-grenoble.fr

Received 11 July 2003

In revised form 27 May 2004

Accepted 9 June 2004

\section{REFERENCES}

1 Benabid AL, Pollak P, Louveau A, et al. Combined (thalamotomy and stimulation) stereotactic surgery of the VIM thalamic nucleus for bilateral Parkinson disease. Appl Neurophysiol 1987:50:344-6.

2 Rehncrona S, Johnels B, Widner H, et al. Long-term efficacy of thalamic deep brain stimulation for tremor: double-blind assessments. Mov Disord 2003; 18:163-70

3 Kumar R, Lozano AM, Sime E, et al. Long-term follow-up of thalamic deep brain stimulation for essential and parkinsonian tremor. Neurology 2003;61:1601-4.

4 Benabid AL, Pollak P, Gervason C, et al. Long-term suppression of tremor by chronic stimulation of the ventral intermediate thalamic nucleus. Lancet 1991;337:403-6.

5 Hirai T, Miyazaki M, Nakajima H, et al. The correlation between tremor characteristics and the predicted volume of effective lesions in stereotaxic nucleus ventralis intermedius thalamotomy. Brain 1983;106:1001-18.

6 Limousin P, Krack P, Pollak P, et al. Electrical stimulation of the subthalamic nucleus in advanced Parkinson's disease. N Engl J Med 1998;339:1 105-11.

7 Krack P, Batir A, Van Blercom N, et al. Five-year follow-up of bilateral stimulation of the subthalamic nucleus in advanced Parkinson's disease. N Engl J Med 2003;349:1925-34.

8 Goetz CG, Leurgans S, Raman R. Placebo-associated improvements in motor function: comparison of subjective and objective sections of the UPDRS in early Parkinson's disease. Mov Disord 2002;17:283-8.

9 Lozano AM, Lang AE, Galvez-Jimenez N, et al. Effect of GPi pallidotomy on motor function in Parkinson's disease. Lancet 1995;346:1383-7.

10 de Boer AG, Wiiker W, Speelman JD, et al. Quality of life in patients with Parkinson's disease: development of a questionnaire. J Neurol Neurosurg Psychiatry 1996;61:70-4

11 Ardouin C, Pillon B, Peiffer E, et al. Bilateral subthalamic or pallidal stimulation for Parkinson's disease affects neither memory nor executive functions: a consecutive series of 62 patients. Ann Neurol 1999;46:217-23.

12 Koller WC, Lyons KE, Wilkinson SB, et al. Long-term safety and efficacy of unilateral deep brain stimulation of the thalamus in essential tremor. Mov Disord 2001;16:464-8

13 Limousin P, Speelman JD, Gielen F, et al. Multicentre European study of thalamic stimulation in parkinsonian and essential tremor. J Neurol Neurosurg Psychiatry 1999;66:289-96.

14 Lyons KE, Koller WC, Wilkinson SB, et al. Long term safety and efficacy of unilateral deep brain stimulation of the thalamus for parkinsonian tremor. J Neurol Neurosurg Psychiatry 2001;71:682-4.

15 Ondo W, Dat Vuong K, Almaguer $M$, et al. Thalamic deep brain stimulation: effects on the nontarget limbs. Mov Disord 2001;16:1137-42.

16 Hariz GM, Bergenheim AT, Hariz MI, et al. Assessment of ability/disability in patients treated with chronic thalamic stimulation for tremor. Mov Disord 1998; 13:78-83.

17 Schuurman PR, Bosch DA, Bossuyt PM, et al. A comparison of continuous thalamic stimulation and thalamotomy for suppression of severe tremor. N Engl J Med 2000;342:461-8.

18 Just $\mathrm{H}$, Ostergaard K. Health-related quality of life in patients with advanced Parkinson's disease treated with deep brain stimulation of the subthalamic nuclei. Mov Disord 2002; 17:539-45.

19 Lagrange $E$, Krack $P$, Moro $E$, et al. Bilateral subthalamic nucleus stimulation improves health-related quality of life in PD. Neurology 2002;59:1976-8.

20 Moro E, Scerrati M, Romito LM, et al. Chronic subthalamic nucleus stimulation reduces medication requirements in Parkinson's disease. Neurology 1999:53:85-90.

21 Krack P, Benazzouz A, Pollak P, et al. Treatment of tremor in Parkinson's disease by subthalamic nucleus stimulation. Mov Disord 1998;13:907-14.

22 Rodriguez MC, Guridi OJ, Alvarez L, et al. The subthalamic nucleus and tremor in Parkinson's disease. Mov Disord 1998:13(Suppl 3):111-18.

23 Beijani BP, Gervais D, Arnulf I, et al. Axial parkinsonian symptoms can be improved: the role of levodopa and bilateral subthalamic stimulation. J Neurol Neurosurg Psychiatry 2000;68:595-600.

24 Gentil M, Garcia-Ruiz P, Pollak P, et al. Effect of stimulation of the subthalamic nucleus on oral control of patients with parkinsonism. J Neurol Neurosurg Psychiatry 1999;67:329-33.

25 Hoveto JL, Beijani PB, Damier P, et al. Failure of long-term pallidal stimulation corrected by subthalamic stimulation in PD. Neurology 2000;55:728-30.

26 Moro E, Esselink RA, Van Blercom N, et al. Bilateral subthalamic nucleus stimulation in a parkinsonian patient with previous unilateral pallidotomy and thalamotomy. Mov Disord 2000; 15:753-5.

27 Pillon B, Ardouin C, Damier P, et al. Neuropsychological changes between "off" and "on" STN or GPi stimulation in Parkinson's disease. Neurology 2000:55:411-18.

28 Saint-Cyr JA, Trepanier LL, Kumar R, et al. Neuropsychological consequences of chronic bilateral stimulation of the subthalamic nucleus in Parkinson's disease. Brain 2000;123:2091-108. 